\title{
Análisis del uso de estrategias complejas de personas con discapacidad intelectual en un contexto de visoconstrucción
}

\author{
Analysis of complex strategies usage in persons with intellectual \\ disability in a visuoconstruction context
}

\begin{abstract}
Resumen
El objetivo del estudio fue valorar la capacidad de planificación, en un contexto de visoconstrucción, de un grupo de personas con discapacidad intelectual. Los 70 participantes eran miembros de Aura Fundación diagnosticados con discapacidad intelectual moderada y fueron reclutados mediante un muestreo intencional. Se haIlaron correlaciones significativas entre el cociente intelectual no verbal, evaluado mediante las Matrices Progresivas de Raven, y la riqueza y exactitud del dibujo de la Figura Compleja de Rey-Osterrieth, así como con el tipo de construcción de la figura. Se constató que los participantes que obtuvieron una mayor puntuación de cociente intelectual llevaron a cabo una mejor planificación de la tarea. También se encontró una correlación significativa entre la riqueza y exactitud de la Figura Compleja de Rey-Osterrieth y el tipo de construcción usado. Estos resultados acreditan la importancia de un sistema de apoyos que impulse el uso de estrategias complejas para lograr objetivos.
\end{abstract}

\section{Palabras clave}

Discapacidad intelectual, sistema de apoyos, visoconstrucción, planificación, Figura Compleja de Rey-Osterrieth.

\begin{abstract}
The goal of the study was to assess planning ability, in a visuoconstruction context, of a group of people with intellectual disability. The 70 participants were members of Aura Foundation diagnosed with moderate intellectual disability and were recruited by intentional sampling. Significant correlations were found between the nonverbal intellectual quotient, evaluated using the Raven Progressive Matrices, and the richness and accuracy of the Rey Figure drawing and between the nonverbal intellectual quotient and the type of creation of the figure. Participants with higher intellectual quotient scores were found to perform better task planning. A significant correlation was also found between the richness and accuracy of the Rey Figure and the type of creation used. These results demonstrate the importance of a support system that impulses the usage of complex strategies to achieve goals.
\end{abstract}

\section{Keywords}

Intellectual disability, system of supports, visuoconstruction, planning, Rey figure.

\author{
Jan Ivern Sala \\ <janis@blanquerna.url.edu> \\ Universitat Ramon Llull. España \\ Xavier Carbonell Sánchez \\ <xaviercs@blanquerna.url.edu> \\ Universitat Ramon Llull. España
}

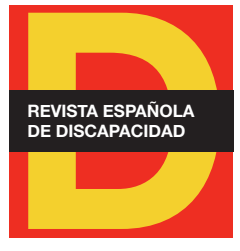

Para citar:

Ivern, J. y Carbonell, X. (2021). Análisis del uso de estrategias complejas de personas con discapacidad intelectual en un contexto de visoconstrucción. Revista Española de Discapacidad, 9(2), pp. 135-152.

Doi: <https://doi.org/10.5569/23405104.09.02.08>

Fecha de recepción: 17-02-2021 Fecha de aceptación: 03-12-2021 


\section{Introducción ${ }^{1}$}

De acuerdo con la American Association on Intellectual and Developmental Disabilities, la discapacidad intelectual se caracteriza por limitaciones significativas tanto en el funcionamiento intelectual como en el comportamiento adaptativo, que se expresan en las habilidades conceptuales, sociales y de adaptación práctica antes de los 22 años (Schalock et al., 2021, p. 1). La prevalencia global en población general es de aproximadamente el 1-3\% (Maulik et al., 2011). El concepto discapacidad intelectual se refiere a los mismos individuos anteriormente diagnosticados de retraso mental (Schalock et al., 2007). Mientras el retraso mental sitúa el foco en la patología o defecto de la persona, la discapacidad intelectual se centra en la comprensión del funcionamiento humano y la discapacidad en base a la interacción entre las características de la persona y las ambientales (Buntinx y Schalock, 2010).

Esta perspectiva socio-ecológica de la discapacidad se focaliza en la calidad de vida de la persona y en la planificación de apoyos individualizados, con el objetivo de potenciar los resultados del funcionamiento humano de la población con discapacidad intelectual. El aumento de su calidad de vida es posible con un sistema de apoyos moldeado e individualizado en función de sus características y necesidades (Wehmeyer et al., 2008; Schalock y Luckasson, 2013). El objetivo de este planteamiento es mejorar los resultados en los dominios del funcionamiento humano que incluyen participación, inclusión, autonomía, independencia, capacidad de decisión, bienestar físico, bienestar mental y desarrollo personal (Buntinx y Schalock, 2010). El sistema de apoyos de referencia es la base sobre la que organizar los apoyos individualizados para potenciar los resultados del funcionamiento de la persona con discapacidad intelectual (Shogren et al., 2014) (ver figura 1).

En el funcionamiento de una persona con discapacidad intelectual todas las dimensiones son susceptibles de alteraciones importantes. Buntinx y Schalock (2010) plantean que la mejora de los resultados es posible a través de un modelo de apoyos de referencia que sirva como base para favorecer la calidad de vida del individuo. En el caso del funcionamiento intelectual, el diseño de los apoyos parte del grado de inteligencia de la persona, que se interpreta mediante una puntuación de cociente intelectual, contextualizada por la experiencia y valoración clínica. Para el diagnóstico de discapacidad intelectual, el criterio que determina las limitaciones significativas en el funcionamiento intelectual es una puntuación de cociente intelectual aproximadamente 2 desviaciones estándar o más por debajo de la media (considerando el error estándar de medida del instrumento específico administrado individualmente) (Schalock et al., 2021, p. 29). El análisis del funcionamiento intelectual es más preciso si se desglosa en dominios cognitivos tales como la capacidad visoespacial y el funcionamiento ejecutivo (Bigelow y Agrawal, 2015). El rendimiento satisfactorio en las tareas cotidianas, fundamento del sistema de apoyos, requiere de la actividad holística de los distintos dominios cognitivos.

1. Agradecimientos: este estudio ha sido posible gracias a Aura Fundación y particularmente a Roser Fernández Olaria, que han cedido los datos necesarios para el desarrollo. 


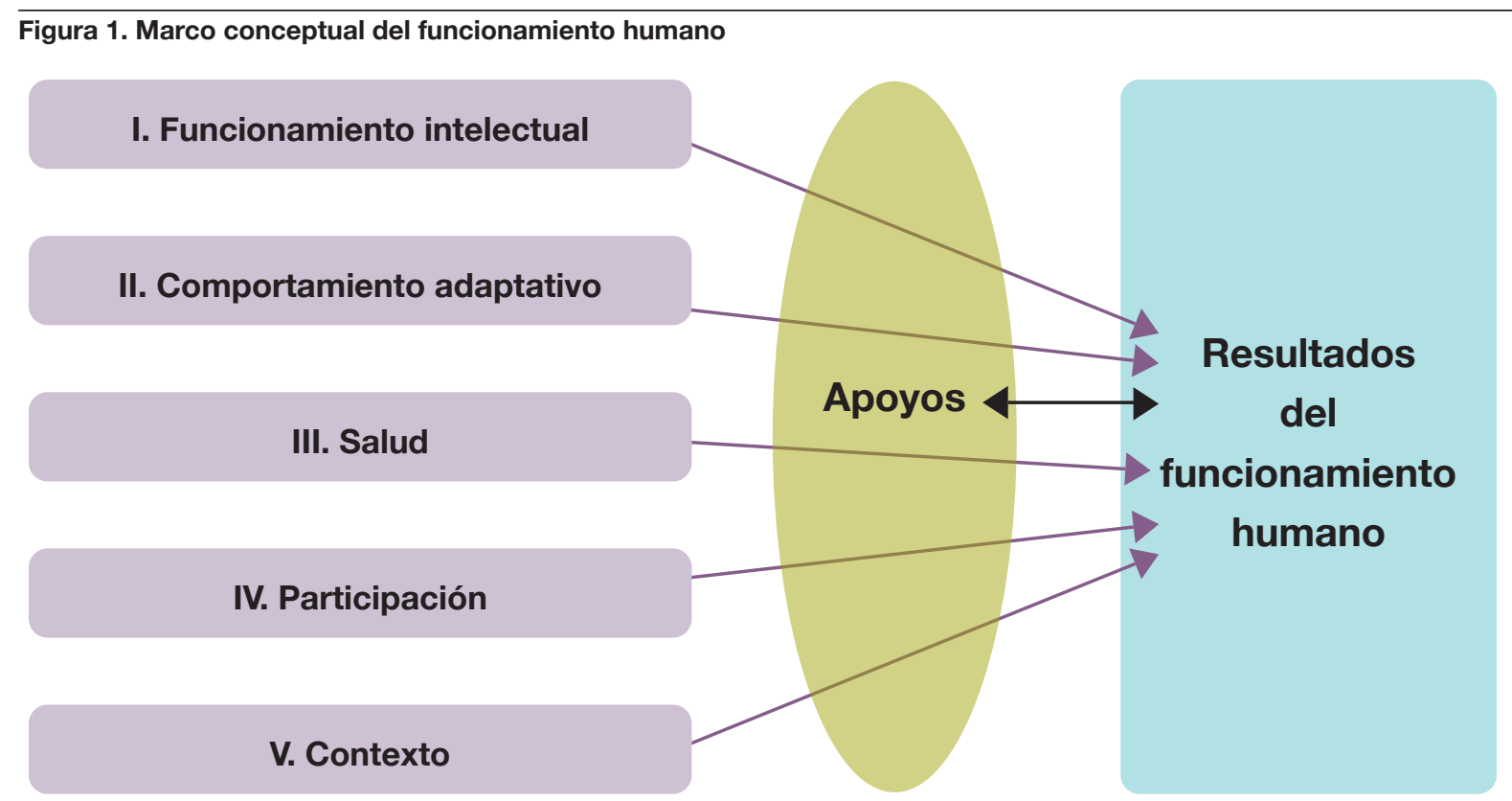

Fuente: Schalock et al., 2021, p. 10.

La individualización de los apoyos en función de las características de cada persona ha abierto el debate del futuro de la investigación en el campo de la discapacidad intelectual. La tradicional concepción de la discapacidad como una disfunción global de los dominios cognitivos se está desmoronando ante las conclusiones de la investigación más reciente, que apunta a una desproporcionalidad significativa entre las fortalezas y debilidades en función de la etiología (Harris et al. 2019). El camino hacia la individualización ha trasladado el foco de la homogeneidad entre personas con discapacidad intelectual a la heterogeneidad según la causa de la discapacidad. Por ejemplo, las personas con síndrome de Williams y síndrome de Kabuki tienen peor habilidad visoespacial y mejores habilidades de lenguaje que las personas con discapacidad intelectual sin síndrome de Williams con equivalente cociente intelectual (Harris et al., 2019; Mervis et al., 2000; Landau y Ferrara, 2013). En cuanto al perfil cognitivo específico de las personas con síndrome de Down, la literatura ha situado las habilidades no verbales, tales como la visoespacialidad y la motivación social, como puntos fuertes y las habilidades de lenguaje y funciones ejecutivas como las áreas más vulnerables de su funcionamiento. (Lecas et al., 2011; Loveall et al., 2017; Rowe et al. 2006). A nivel de funcionamiento ejecutivo, las personas con síndrome de Down presentan un rendimiento óptimo en tareas simples y concretas y un empeoramiento brusco en tareas complejas que demandan integración (Grieco et al., 2015).

Los escasos programas cognitivos de funciones ejecutivas que se elaboran se destinan al síndrome de Down y sitúan el foco en las funciones ejecutivas de nivel bajo (debido a su disfuncionalidad ejecutiva básica vinculada a alteraciones morfológicas del lóbulo frontal) y en las habilidades de lenguaje por ser consideradas su gran debilidad (Grieco et al., 2015; Loveall et al., 2017). Estudiar la planificación visoespacial de personas con discapacidad intelectual, excluyendo a aquellos perfiles cognitivos que por su naturaleza tienen un deterioro de las habilidades visoespaciales o ejecutivas (síndrome de Down, de Williams y de Kabuki), representa una 
disminución del sesgo de la muestra y es preciso para alcanzar el potencial de aprendizaje de perfiles cognitivos más difíciles de estudiar a nivel metodológico como la discapacidad intelectual de etiología indeterminada. La planificación evoluciona sustancialmente durante la infancia tardía y la adolescencia, por lo que se debe ser extremadamente prudente al extraer conclusiones prematuras respecto a la capacidad de planificación sobre participantes menores de 18 años, especialmente en un campo como la discapacidad intelectual (Ferguson et al., 2021). Por consiguiente, la presente investigación ha sido basada en adultos jóvenes.

Multitud de labores del día a día tales como dibujar, hacer la cama o atarse los botones de la camisa son posibles gracias a la habilidad visoconstructiva. La visoconstrucción es una función cognitiva central que posibilita visualizar un modelo como un conjunto de partes y reproducir su estructura a partir de sus componentes. Su evaluación suele basarse en el análisis semicuantitativo de la reproducción con papel y lápiz de una figura compleja (Nielsen y Jørgensen, 2013). El proceso requiere analizar los elementos del modelo y sus proporciones visoespaciales, plantear un plan de construcción estableciendo una secuencia y jerarquía de los componentes de forma lógica y ejecutarlo a nivel motor (Mervis et al., 1999). Para realizar procesos visoconstruccionales es necesario formar representaciones mentales de los objetos, formas y sus posiciones y mantener esa información de una forma que haga posible manipularla mentalmente (Nguyen et al., 2014). Este punto representa el auge de la interconectividad entre las habilidades visoespaciales y las funciones ejecutivas. En otras palabras, refleja como la visoespacialidad precede al funcionamiento ejecutivo y es fundamental para su desarrollo (Osmon et al., 2006). En este sentido, es necesario un cierto nivel de su competencia para desarrollar procesos ejecutivos de mayor complejidad como los implicados en la reproducción de una figura compleja (Assel et al., 2003). Aunque los déficits en las funciones ejecutivas son frecuentemente descritos en trastornos del desarrollo como la discapacidad intelectual, su evaluación en esta población no es una práctica habitual (Costanzo et al., 2013).

Las funciones ejecutivas (inhibición de la respuesta, memoria de trabajo y flexibilidad cognitiva) son funciones mentales superiores que, junto a otros procesos mentales de menor nivel, permiten al individuo regular pensamientos y acciones para llevar a cabo objetivos de forma efectiva (Friedman y Miyake, 2017; Miyake et al., 2000). El rendimiento satisfactorio en tareas visoconstructivas suele estar vinculado a una buena planificación, formulación de objetivos, iniciativa y capacidad de hacer actividades que requieren el uso de estrategias complejas para la consecución de las metas (Lezak et al., 2004, pp. 253, 624). La afectación de estas capacidades en discapacidad intelectual suele implicar mayor dificultad para resolver problemas, secuenciación de subobjetivos incoherente, desorganización, dificultades para desarrollar estrategias eficientes y dependencia de estrategias previamente aprendidas (Anderson, 2002). El impacto que el funcionamiento ejecutivo tiene en los resultados del funcionamiento humano evidencia la necesidad de priorizar su entrenamiento y seguimiento desde el sistema de apoyos. Por ejemplo, la capacidad para cambiar flexiblemente de tarea es clave para el comportamiento adaptativo, controlar el impulso que lleva a alguien a comer peor o más de lo necesario tiene consecuencias en su salud, la inhibición de respuestas prepotentes es necesaria para participar satisfactoriamente en el ámbito social y la regulación de la conducta es imprescindible para un correcto proceso de aprendizaje en el contexto escolar (Miyake y Friedman, 2012). La planificación, formulación de objetivos, iniciativa y capacidad de llevar a cabo una actividad compleja para lograr los objetivos contribuyen a la consecución satisfactoria de tareas visoconstructivas (Lezak et al., 2004, p. 624). Específicamente, la precariedad de la visoconstrucción está vinculada a un deterioro de la planificación (Lezak et al., 2004, p. 253).

Barroso y León (2002) definieron la planificación como la capacidad para plantear objetivos, establecer ensayos mentales, aplicar las estrategias deseadas y valorar el grado en el que se han logrado los objetivos 
pretendidos y las consecuencias. La planificación requiere de la actividad holística de las funciones ejecutivas, consideradas el pilar fundamental de la consecución de objetivos. Para planificar es necesario formular subobjetivos, es decir, organizar el proceso en pasos específicos coherentemente secuenciados para lograr el objetivo final. Una vez definido el subobjetivo, se diseña la conducta para llevarlo a cabo con el fin de anticipar las consecuencias (Hung et al., 2013). Un alto grado de flexibilidad cognitiva facilita los posibles cambios necesarios entre subobjetivos a medida que el plan avanza (Pellicano, 2010). Gracias a este proceso es posible enfocar una tarea visoconstructiva de manera organizada, estratégica y eficiente (Pennequin et al., 2010). Hoy en día la literatura sigue sin ofrecer una imagen clara de la planificación en personas con discapacidad intelectual (Danielsson et al., 2010). La disparidad entre los resultados obtenidos por las distintas investigaciones se traduce en una incapacidad para extraer conclusiones sólidas sobre la capacidad de planificar de la población con discapacidad intelectual. Este fenómeno supone un impedimento para enfocar eficazmente el tratamiento de la planificación desde el modelo de apoyos (Palmqvist et al., 2020). En el campo de la discapacidad intelectual, la Torre de Londres y la Torre de Hanoi han sido los test más usados para el estudio de la planificación a corto plazo (Shallice, 1982). Son pruebas de resolución de problemas y planificación espacial donde el objetivo del examinado es copiar un diseño realizando el mínimo de movimientos posibles. Aunque los déficits en las funciones ejecutivas son frecuentemente descritos en trastornos del desarrollo como la discapacidad intelectual, la evaluación de la función ejecutiva en personas con discapacidad intelectual no es una práctica habitual (Costanzo et al., 2013). Muy pocos test evalúan la planificación per se y la escasez es aún mayor en el campo de la discapacidad intelectual (Lezak et al., 2004, p. 614). En el caso de la Torre de Londres y la Torre de Hanoi, la planificación implicada está directamente condicionada por la inhibición de respuestas prepotentes (Lezak et al., 2004, p. 619; Miyake et al., 2000).

Danielsson et al. (2010) no encontraron diferencias significativas entre el rendimiento en la Torre de Hanoi de un grupo de personas con discapacidad intelectual respecto a un grupo comparativo. Ante tal hallazgo, concluyeron que las estrategias simples requeridas en la Torre de Hanoi (acciones que perceptiblemente acercan al objetivo sin necesidad de plantear subobjetivos) demandan menos capacidad cognitiva y su uso es mucho más frecuente y automático. Masson et al. (2010) observaron una correlación positiva entre el cociente intelectual y la puntuación en la Torre de Londres en individuos con discapacidad intelectual con etiología variada. Observaron que el rendimiento de las personas con discapacidad intelectual no seguía el mismo patrón que el de la población general; descendían de forma mucho menos gradual conforme el nivel de dificultad aumentaba. Había un empeoramiento brusco de los resultados entre la tarea más asequible de las que involucraban pasos orientados a subobjetivos (estrategias complejas) y la menos asequible de las que no (estrategias simples).

El objetivo de esta investigación ha sido analizar el uso de estrategias complejas para la resolución de problemas (que involucran pasos orientados a subobjetivos), dentro de un contexto de visoconstrucción, de un grupo de personas con discapacidad intelectual. La actividad holística de las funciones ejecutivas es necesaria para la resolución de las tareas espaciales más difíciles de la Torre de Londres, pero su correlación con los resultados en los problemas sencillos es mucho menos significativa (Dias y Seabra, 2012). Este elemento distintivo supone un obstáculo para la planificación de las personas con discapacidad intelectual tal y como describe el estudio de Masson et al. (2010). En esta línea, es necesario indagar en el análisis del uso de estrategias complejas, como las implicadas en la reproducción de una figura compleja, para acercarnos más al potencial de aprendizaje de la población con discapacidad intelectual. La planificación evoluciona sustancialmente durante la infancia tardía y la adolescencia, por lo que se debe ser extremadamente prudente al extraer conclusiones prematuras respecto a la capacidad de planificar de personas menores de 18 años, 
especialmente en un campo como la discapacidad intelectual (Ferguson et al., 2021). Por consiguiente, la presente investigación ha sido basada en adultos jóvenes (entre 19 y 33 años).

El presente artículo es una réplica del análisis de la planificación de Castañeda et al. (2017) que realizaron como parte de una evaluación neuropsicológica del funcionamiento ejecutivo en adultos jóvenes (entre 20 y 39 años) con discapacidad intelectual moderada. El estudio concluyó, en base a los resultados del test visoconstructivo de la Figura Compleja de Rey-Osterrieth, que el sexo masculino planificó mejor la tarea que el femenino, que el deterioro de la planificación de las personas con discapacidad intelectual moderada es significativo y que la calidad de esta estaba influenciada por la comprensión y respeto de las instrucciones. Sin embargo, el análisis de Castañeda et al. (2017) tenía 3 limitaciones importantes que cuestionan la validez de estas conclusiones y que han sido consideradas en el presente estudio: la muestra de hombres y mujeres era poco significativa y presentaba un sesgo excesivo, prácticamente no se analizaron cualitativamente los resultados y no se estudió la relación entre la planificación y la capacidad de razonamiento de los participantes. La evaluación neuropsicológica es necesaria para seguir mejorando el sistema de apoyos que se ofrece al colectivo y, por este motivo, la presente investigación aporta conclusiones relevantes para el avance en el campo de la discapacidad intelectual.

\section{Método}

\subsection{Participantes}

Participaron 70 personas con diagnóstico de discapacidad intelectual sin síndrome de Down de Aura Fundación (Barcelona). 36 fueron hombres $(51,42 \%)$ y 34 mujeres $(48,57 \%)$, todos y todas mayores de edad. La edad osciló entre los 19 y 33 años (media=23,53, DT=2,98), los hombres 22,69 años (DT=2,63) y las mujeres 24,41 años (DT=3,10). Formaban parte de un programa de funcionamiento adaptativo donde se lleva a cabo formación e impulsión de la autonomía personal, inclusión laboral, y seguimiento laboral y personal.

Los participantes tenían un grado de discapacidad intelectual moderado según la escala de gravedad de la discapacidad intelectual del DSM-5 (APA, 2014, pp. 34-35). De los 99 miembros que llevaron a cabo el screening de evaluación cognitiva de Aura Fundación se seleccionaron todos para participar excepto 29 que presentaban alguna de estas 7 características: (1) grado de discapacidad grave o profundo, (2) diagnóstico de síndrome de Down, (3) diagnóstico de síndrome de Williams, (4) diagnóstico de síndrome de Kabuki, (5) incapacidad para comprender las tareas, (6) afectaciones visuales o (7) afectaciones del funcionamiento motor.

\subsection{Instrumentos}

Para el cociente intelectual se utilizó el test de las Matrices Progresivas de Color (CPM) (Raven et al., 2001). Esta prueba evalúa la capacidad para comparar formas y razonar no verbalmente, con independencia de los conocimientos adquiridos de acuerdo al contexto cultural de la persona. Están diseñadas para niños (de 
entre 5,5 y 11 años) y adultos con discapacidad intelectual. Se seleccionó porqué se enfoca en las funciones visoespaciales y la lógica desde una perspectiva no verbal, lo cual resulta conveniente para estudiar posibles correlaciones entre cociente intelectual y desempeño en la tarea visoconstructiva (Luzzi et al., 2011).

El test se compone de tres series de 12 dibujos cada una representadas en un cuaderno. Los 36 ítems están ordenados en complejidad creciente y en cada serie siguen una lógica diferente. Los ítems se presentan como patrones de colores con una porción faltante y 6 posibles opciones para rellenarla. La ejecución requiere habilidad para completar un patrón continuo, percepción de las partes de un patrón como un todo en base a relaciones espaciales y razonamiento analógico (Facon y Nuchadee, 2010).

Para la planificación visoespacial se utilizó el Test de copia de una Figura Compleja de Rey-Osterrieth (CFCR) (Rey, 2009). Esta prueba evalúa habilidad visoespacial, funciones ejecutivas, psicomotricidad, memoria visual inmediata y atención. Se pidió a los participantes que copiaran la figura con el modelo a la vista (figura 2).

\section{Figura 2. Modelo de la figura compleja de Rey-Osterrieth}

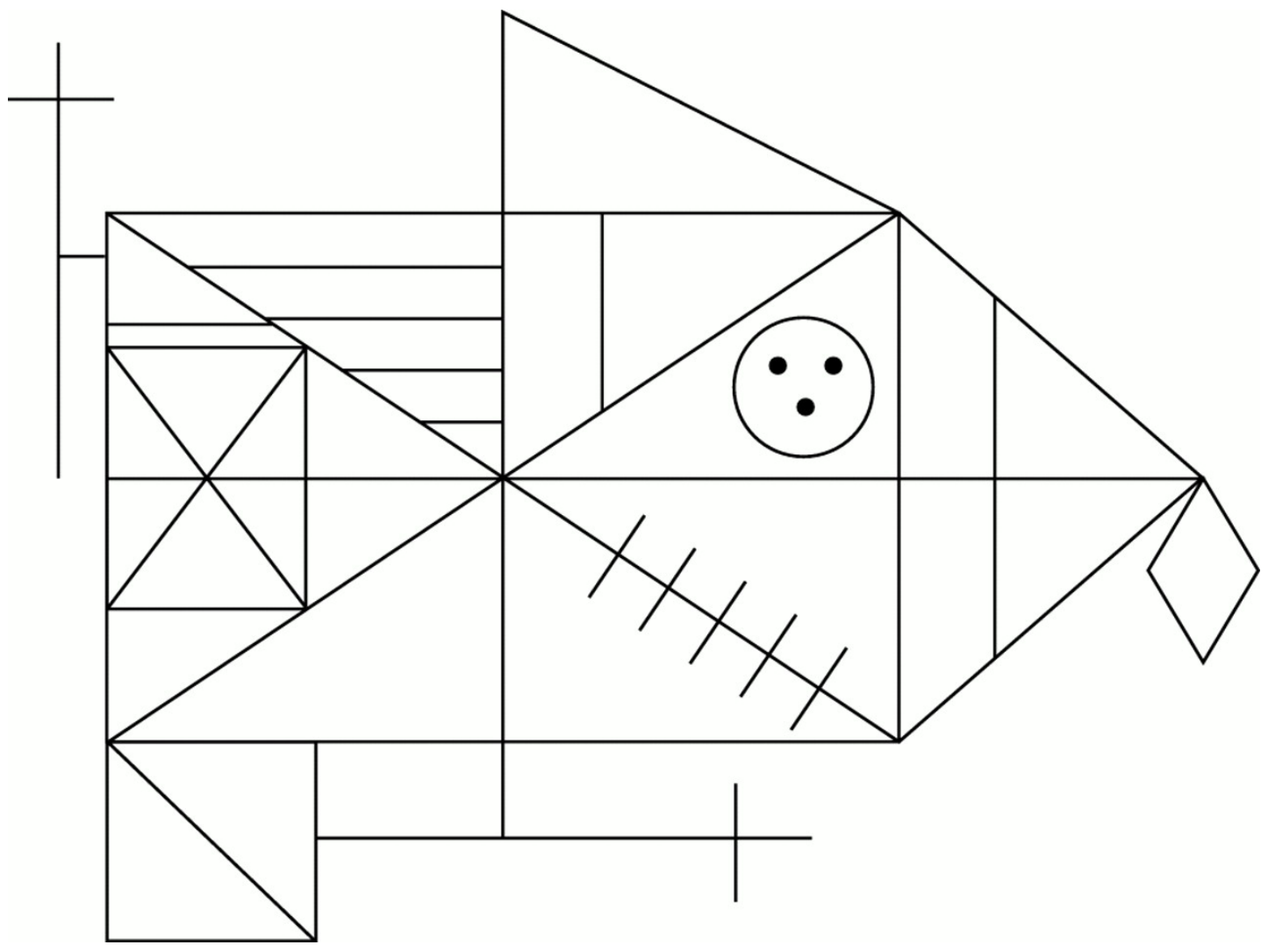

Fuente: Rey, 2009. 
Se interpretó cuantitativamente el resultado en base al sistema de puntuación de Meyers y Meyers (1995), método que divide la figura en 18 elementos y los evalúa en base su exactitud y riqueza. El tiempo de ejecución se cuantificó con un cronómetro y no se estableció un tiempo límite. Se interpretó cualitativamente el procedimiento en base al sistema de puntuación de Osterrieth $(1944,1993)$, que analiza la eficiencia de las estrategias usadas (los elementos con que se inicia la copia, el dibujo del rectángulo separado de los detalles y si se enfoca el dibujo pieza por pieza o como un todo) (Troyer y Wishart, 1997). Se ha definido una escala categórica de tipos de construcción ordenados del primero al séptimo según su grado de nivel racional (ver tabla 1$)$.

\begin{tabular}{|c|c|}
\hline Eficiente & No eficiente \\
\hline $\begin{array}{l}\text { I.) Empezar por el rectángulo principal, añadir detalles en } \\
\text { relación a la totalidad de la figura. }\end{array}$ & $\begin{array}{l}\text { IV.) Dibujar subcomponentes específicos sin una } \\
\text { organización clara. }\end{array}$ \\
\hline $\begin{array}{l}\text { II.) Empezar por un detalle o subcomponente, terminar el } \\
\text { rectángulo y añadir detalles. }\end{array}$ & V.) Dibujar detalles sin una estructura organizacional. \\
\hline \multirow[t]{2}{*}{$\begin{array}{l}\text { III.) Empezar por el contorno de la figura, añadir detalles de } \\
\text { arriba abajo o de izquierda a derecha. }\end{array}$} & VI.) Dibujar un objeto similar (barco, cohete o casa). \\
\hline & VII.) Dibujar garabatos. \\
\hline
\end{tabular}

Fuente: Osterrieth, 1944, 1993.

\subsection{Procedimiento}

Este es un estudio observacional transversal analítico con muestreo intencional para la selección de participantes. La administración de las pruebas cognitivas, su corrección y posteriormente la recopilación de datos se llevaron a cabo en las instalaciones del centro de Aura Fundación. Las pruebas se llevaron a cabo en una sala con silencio, al lado de donde los participantes realizan habitualmente las actividades grupales de formación y seguimiento laboral. La primera lengua de los participantes era el catalán o el castellano, por lo que las pruebas se administraron en una u otra según el caso. Aura Fundación es una entidad sin ánimo de lucro creada en 1989 que ofrece servicios para la inclusión social y laboral de personas con discapacidad intelectual siguiendo la metodología "Empleo con apoyo", basada en la teoría del sistema de apoyos de la American Association on Intellectual and Developmental Disabilities (AAIDD).

Las Matrices Progresivas de Color y la Figura Compleja de Rey-Osterrieth forman parte de un screening de evaluación cognitiva diseñado por Aura Fundación específicamente para personas adultas con discapacidad intelectual. Se administró en unos 60 minutos, aunque varió notablemente según las necesidades del participante. En esta línea, la administración se hizo en 1 día o se dividió en 2 en función de cada caso, con el fin de asegurar el rendimiento óptimo de los examinados. El screening se utiliza para el seguimiento neuropsicológico de los usuarios de la fundación y valora múltiples procesos cognitivos (atención, memoria, velocidad de procesamiento, funcionamiento ejecutivo, razonamiento verbal y razonamiento no verbal). No suele utilizarse en personas con síndrome de Down, población para el que han diseñado un screening de evaluación cognitiva específico. 
Aura Fundación cedió los datos necesarios para el desarrollo de este estudio retrospectivo, contando con el consentimiento informado de los clientes.

\subsection{Análisis de datos}

Para el tratamiento de los datos recogidos se utilizó el paquete estadístico IBM SPSS Statistics versión 25.0. Mediante la prueba de Kolmogorov-Smirnov se analizó la normalidad de los datos de la muestra. Se concluyó que ninguna variable seguía una distribución normal a excepción del cociente intelectual, medido a través de las puntuaciones directas de las Matrices Progresivas de Color. Por tanto, para relacionar el cociente intelectual (Matrices Progresivas de Color) con la planificación visoespacial (Figura Compleja de Rey-Osterrieth) y los criterios para la interpretación de los resultados de la Figura Compleja de Rey-Osterrieth entre ellos (exactitud y riqueza de la copia, tiempo de ejecución y tipo de construcción de la figura) se utilizaron correlaciones de Spearman.

Se analizaron posibles diferencias significativas a nivel de sexo del modo siguiente: la exactitud y riqueza de la Figura Compleja de Rey-Osterrieth a través de la prueba no paramétrica U-Mann Whitney, el tiempo de ejecución de la Figura Compleja de Rey-Osterrieth también con la U-Mann Whitney, el cociente intelectual (Matrices Progresivas de Color) mediante la prueba paramétrica T-Student y el tipo de construcción de la Figura Compleja de Rey-Osterrieth con la prueba no paramétrica Chi-cuadrado de Pearson $\left(\mathrm{X}^{2}\right)$.

El tratamiento de datos se llevó a cabo manteniendo las puntuaciones directas, tanto en el caso de las Matrices Progresivas de Color como en las escalas cuantitativas de la Figura Compleja de Rey-Osterrieth. Se utilizaron puntuaciones directas y no percentiles para discriminar mejor las diferencias entre participantes. Sería perjudicial transformar las puntuaciones directas en base a unos baremos que solo presentan los puntos centiles más importantes (para no ser excesivamente precisos) que no están adaptados para personas con discapacidad intelectual. Además, se da la conveniencia de que el rango de las puntuaciones directas es de 36 en ambas pruebas.

\section{Resultados}

Los 70 participantes obtuvieron una puntuación directa media en las Matrices Progresivas de Color de 24,78 sobre 36 (DT=6,26). En cuanto a la Figura Compleja de Rey-Osterrieth, la puntuación directa media fue de 24,76 sobre 36 (DT=9,09), tardando una media de 282,67 segundos (DT=153,72) y construyendo la figura según se indica en la figura 3. 


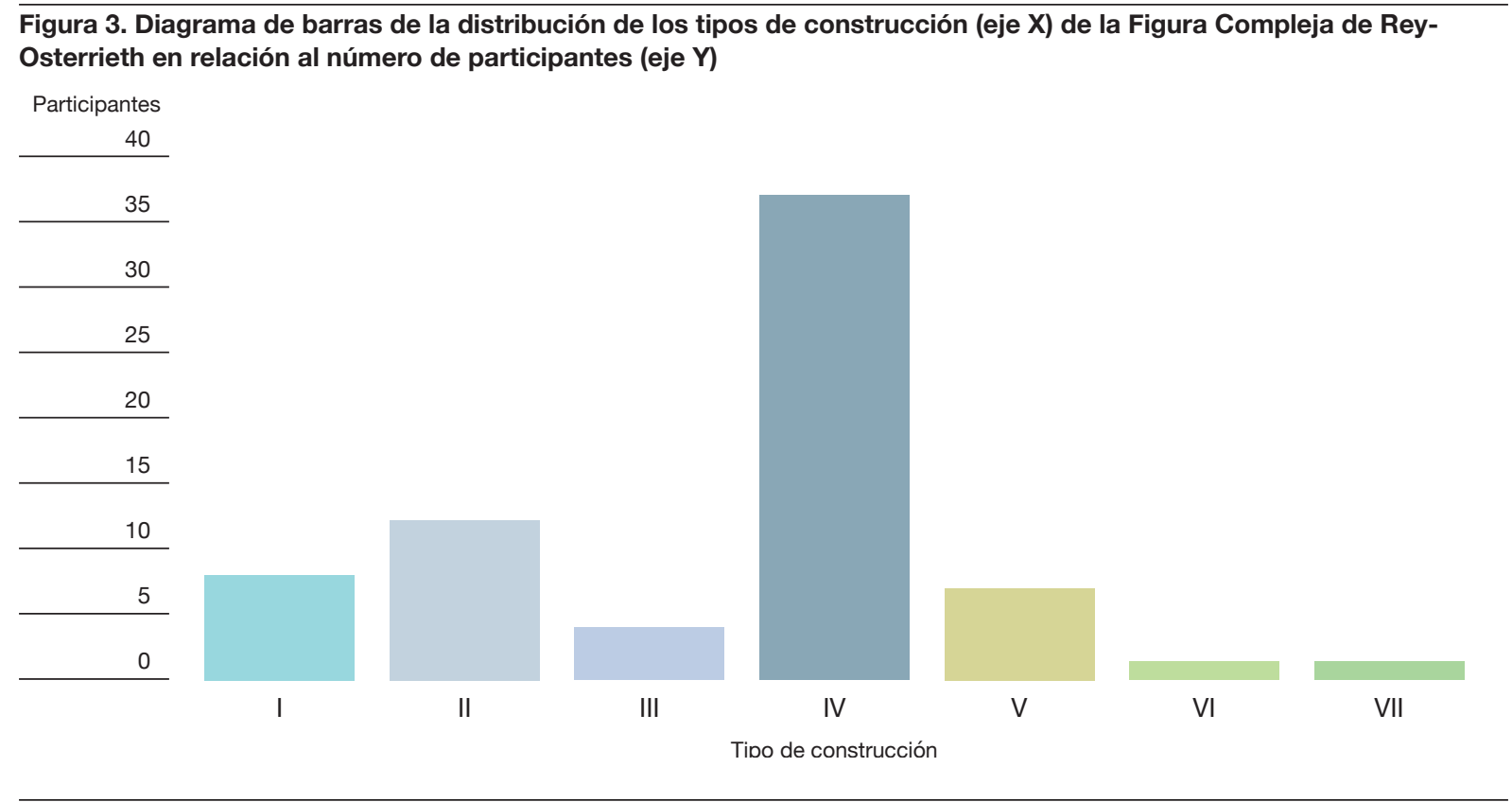

En cuanto a las diferencias entre sexos, en ningún caso resultaron estadísticamente significativas. No lo fueron por lo que respecta al cociente intelectual $(p=.33)$, ni a la riqueza y exactitud de la Figura Compleja de Rey-Osterrieth $(p=.61)$, ni al tiempo de ejecución $(p=.34)$ ni al tipo de construcción $(p=.11)$.

Se calcularon las correlaciones entre el cociente intelectual (puntuaciones directas de las Matrices Progresivas de Color) y la planificación (exactitud y riqueza, tiempo de ejecución y tipo de construcción de la copia de la Figura Compleja de Rey-Osterrieth) (ver tabla 2). Entre el cociente intelectual y la riqueza y exactitud de la copia se encontró una correlación positiva estadísticamente significativa $(r=.765, p=.01)$. Entre el cociente intelectual y el tipo de construcción de la figura también se halló una correlación significativa $(r=-.512$, $p=.01)$. El valor de $r$ es negativo ya que el tipo de construcción I es el que requiere un mayor nivel racional y el tipo VII el que menos y así se ha mantenido durante el tratamiento de los datos. No se encontraron correlaciones estadísticamente significativas entre el cociente intelectual y el tiempo de ejecución ( $r=-.019$, $p=.87)$.

También se calcularon las correlaciones de los criterios de evaluación de la Figura Compleja de Rey-Osterrieth entre ellos. Se halló una correlación significativa entre la riqueza y exactitud de la figura y el tipo de construcción $(r=-.638, p=.01)$. Entre el tipo de construcción y el tiempo de ejecución la correlación no resultó significativa $(r=.16, p=.17)$. Finalmente, la correlación entre la riqueza y exactitud de la figura y el tiempo de ejecución tampoco fue significativa $(r=.075, p=.53)$. 


\begin{tabular}{|c|c|c|c|}
\hline & CFCR- Riqueza y exactitud & CFCR- Tiempo de ejecución & CFCR- Tipo de construcción \\
\hline CPM- cociente intelectual & $r=.765^{*}$ & $r=-0.19$ & $r=-.512^{*}$ \\
\hline CFCR- Riqueza y exactitud & & $r=.16$ & $r=-.638^{*}$ \\
\hline CFCR- Tiempo & & & $r=.075$ \\
\hline
\end{tabular}

*significativa.

\section{Discusión}

Este estudio ha analizado el desempeño en la visoconstrucción de una figura compleja de personas con discapacidad intelectual moderada, con el fin de mejorar la evaluación neuropsicológica y mejorar el encauzamiento de los apoyos destinados al seguimiento y entrenamiento del funcionamiento intelectual de esta población. Se ha estudiado la planificación visoespacial en base a la riqueza y exactitud, tiempo de ejecución y tipo de construcción de la Figura Compleja de Rey-Osterrieth y la respectiva observación clínica del procedimiento, así como su relación con el sexo y con las funciones visoespaciales y la lógica no verbal (expresadas vía puntuaciones de cociente intelectual).

No se han encontrado diferencias significativas entre hombres y mujeres por lo que respecta a las puntuaciones de cociente intelectual y el desempeño en la Figura Compleja de Rey-Osterrieth; en consecuencia, se han tratado todos los datos conjuntamente. Este hallazgo contrasta con el rendimiento superior de los hombres respecto las mujeres que encontraron Castañeda et al. (2017). La razón de esta discrepancia podría ser el hecho que lbáñez et al. no tuvieron en cuenta los resultados de aquellos participantes que necesitaron más de 5' para completar la tarea, que fueron más hombres ( $57 \%$ del total de hombres) que mujeres (50\% del total de mujeres). Los resultados de este estudio cuestionan tal criterio, dado que los participantes emplearon de media 4' 43" para reproducir la figura y no se ha encontrado una correlación significativa entre el tiempo empleado y el rendimiento en la tarea. Goyal et al. (2018) también concluyeron que el sexo no era un predictor significante de los procesos implicados en la visoconstrucción, aunque otros autores difieren (Kitler et al., 2004).

Se ha constatado que los participantes que han obtenido una mayor puntuación de cociente intelectual se han desenvuelto de forma más efectiva y eficaz durante la tarea visoconstructiva. Luciana et al. (2009) exploraron específicamente el vínculo entre el cociente intelectual y la planificación en población general y hallaron una correlación muy baja. Según Ardila et al. (2000), no es de extrañar que la correlación entre el cociente intelectual y la planificación no sea concluyente porque los test de inteligencia no son sensibles a procesos metacognitivos como el diseño y ejecución de planes para acercarse a objetivos. Las pobres correlaciones significativas que Ardila et al. (2000) encontraron en población general contrastan con las halladas en nuestra investigación y con las de Masson et al. (2010). En ambos estudios, llevados a cabo con 
personas con discapacidad intelectual, se han encontrado correlaciones positivas muy significativas entre el cociente intelectual de la persona con discapacidad intelectual y la planificación.

Llama la atención que en participantes con discapacidad intelectual la correlación entre el cociente intelectual y la planificación no solo es concluyente, sino que es mucho más significativa que en estudios donde los participantes no tenían discapacidad intelectual. Esto evidencia una especial dificultad de las personas con discapacidad intelectual para planificar. Crear representaciones mentales y diseñar estrategias complejas son procesos mentales de alto nivel que requieren un mayor grado de control, por lo que se muestran especialmente sensibles cuando el razonamiento se sitúa por debajo de la media. La correlación positiva que Masson et al. (2010) encontraron entre el cociente intelectual total y la planificación de la tarea visoespacial fue aún más significativa en este estudio. Como ambas tareas (Figura Compleja de Rey-Osterrieth y Torre de Londres) implican primordialmente funciones no verbales, es coherente que el cociente intelectual total no correlacione tan significativamente con el rendimiento de la tarea como el cociente intelectual específicamente no verbal.

Más de la mitad de los participantes reprodujeron la figura compleja mediante el tipo IV, ensamblando los detalles contiguos unos a otros y procediendo como si construyeran un rompecabezas (Rey, 2009). Este tipo de construcción demuestra una incapacidad de planear una estrategia de producción que resulte en una segmentación de la figura en dos partes. No se lleva a cabo una selección y jerarquización de los elementos que constituyen la figura para secuenciar el proceso de copia de forma lógica (Sommers, 1989). Se ha mostrado una incapacidad para generar un plan moldeable en función de las condiciones específicas de la tarea. Era la primera vez que copiaban el modelo y mayoritariamente no se planearon estrategias adaptadas a ese estímulo nuevo. En consecuencia, construyeron la figura en base a un criterio universal que serviría para la reproducción de cualquier dibujo. Esta incapacidad denota un obstáculo importante para la planificación contingente de las personas con discapacidad intelectual moderada.

Pocos participantes dibujaron la figura con las estrategias más eficientes, empezando por un armazón que sirva de referencia y añadiendo los detalles en función de un orden lógico. También fueron pocos los que construyeron la figura en base a los procedimientos menos eficaces, que generalmente resultan en una copia carente de estructuración en la que los elementos del modelo son muy poco reconocibles. Los participantes que construyeron la figura con mayor nivel de racionalidad dibujaron sus elementos con más riqueza y exactitud. Por el contrario, quienes realizaron un procedimiento más primitivo obtuvieron un resultado de la tarea de copia más pobre; omitiendo o añadiendo elementos, transformándolos o variando sus posiciones. Masson et al. (2010) hallaron que los participantes tenían dificultades en la transición entre un problema de dos movimientos y uno de tres de la Torre de Londres, algo muy parecido a lo sucedido en el presente estudio. El tipo de construcción III ha resultado ser un obstáculo para muchos de los participantes. Hasta el tipo III no se requiere la formulación de subobjetivos ni la consideración de posibles conflictos de movimientos, ya que no existe un elemento director de la reproducción. Una explicación similar fue dada por Masson et al. (2010) para justificar los resultados de su estudio. Danielsson et al. (2012) estudiaron las diferencias entre el perfil cognitivo de personas con discapacidad intelectual y de personas con la edad mental proporcional. Tenían una capacidad similar en cuanto a la fluencia y el cambio de atención entre tareas, pero especial dificultad en la planificación, la inhibición de respuestas prepotentes y la memoria de trabajo. De tal manera, el constructo discapacidad intelectual se aleja aún más del término retraso mental, que entiende la discapacidad como un desajuste entre las capacidades mentales y la edad cronológica y no como otra forma de funcionamiento. Esta investigación corrobora la hipótesis de que la accesibilidad a las funciones 
mentales superiores como la planificación visoespacial es menor en las personas con discapacidad intelectual que los procesos cognitivos más básicos.

Durante la administración se produjo un fenómeno que las observaciones de Castañeda et al. (2017) ya dejaron entrever, una importante dificultad de adaptación a la tarea en general y en especial por parte de aquellos participantes cuyo rendimiento fue peor. A pesar de atender y comprender las instrucciones dadas, tenían conductas poco conscientes con el fin de adaptar la tarea a su comodidad, tales como colocar la hoja en posición vertical cuando se le ha dado en horizontal, hacer el dibujo pequeño o en los extremos de la hoja, cambiar de colores sin indicación, sobreponer demasiado la hoja del modelo a la hoja de la copia o hablar durante el procedimiento. En este sentido, podemos situar la causa de estas conductas en dominios cognitivos como atención, comprensión y funcionamiento ejecutivo y en factores más vinculados a la personalidad como impulsividad y rigidez, pero la causa predominante es difícil de establecer y es muy variable.

Por lo que respecta al tiempo de ejecución no se ha asociado ni con el tipo de construcción ni con la riqueza y exactitud de la copia. En población general, el tiempo de ejecución correlaciona significativamente con el desempeño en la tarea (riqueza y exactitud y tipo de construcción de la copia), siendo peor cuanto más tiempo empleado (Tremblay et al., 2015). Sin embargo, la correlación no ha sido significativa en el presente estudio, donde ha habido una gran variedad de tiempos de copia independientemente del tipo de construcción y del resultado de la tarea. Hay varias posibles explicaciones para este fenómeno, como un mayor impacto de factores de personalidad como la impulsividad o el perfeccionismo y el efecto de una peor planificación de la tarea, que da lugar a tiempos de ejecución más largos y más variables en comparación a la población de edad mental proporcional. La baja correlación entre los resultados de la tarea y el tiempo empleado corrobora la heterogeneidad de perfiles cognitivos en el campo de la discapacidad intelectual en comparación con la población general y la consecuente necesidad de desarrollar la individualización del sistema de apoyos.

En cuanto a las limitaciones de la investigación, no fue posible estudiar la planificación en base a un sistema cualitativo de puntuación que permitiera conocer el orden en que el sujeto copió los distintos segmentos de la Figura Compleja de Rey-Osterrieth. Si bien Osterrieth $(1944,1993)$ diseñó su sistema cualitativo de puntuación con el fin de analizar la planificación y las habilidades organizativas, Anderson et al. (2001) expusieron que este sistema ignora algunas estrategias más variables y menos sistemáticas en comparación con otros sistemas como el Sistema Cualitativo de Boston para la Figura Compleja de Rey-Osterrieth (BQSS) (Stern et al., 1994).

En conclusión, el presente estudio aporta información relevante de cara al planteamiento de la evaluación neuropsicológica en el campo de la discapacidad intelectual. Las diferencias significativas en el rendimiento que encontraron Ibáñez et al. (2017) han sido contradichas y se ha corroborado la dificultad en el funcionamiento mental superior implicado en la visoconstrucción. Se ha profundizado en la dificultad específica en el funcionamiento ejecutivo de nivel alto analizando la planificación en un contexto de visoconstrucción y se ha relacionado con el razonamiento no verbal. Se ha concretado que el uso de estrategias complejas y la consecuente formulación de subobjetivos suponen un obstáculo para el rendimiento favorable y el consecuente acercamiento al potencial de aprendizaje de las personas con discapacidad intelectual moderada. El seguimiento y entrenamiento de la planificación en distintos contextos es clave para el apoyo del funcionamiento intelectual y el contexto de visoconstrucción permite observar con detalle el uso de estrategias complejas.

Las tareas visocontructivas tienen un gran potencial para la evaluación de habilidades superiores como la planificación visoespacial, pero la complejidad del análisis cualitativo es fundamental para sonsacar con- 
clusiones sólidas debido a la gran cantidad de variables que condicionan el rendimiento. En este sentido, el presente estudio ha replicado con mayor precisión el estudio de lbáñez et al. (2017) pero debido a la complejidad de la función que se estudia y de la tarea, es necesario seguir replicándolo e indagando en los aspectos cualitativos y su relación con los cuantitativos. Por ende, de cara a futuras investigaciones, conviene profundizar en la investigación cualitativa de los dibujos para precisar y analizar con lupa los procesos subyacentes. El foco de la investigación debe situarse en las personas que tienen gran dificultad en el planteamiento de estrategias complejas para copiar la Figura Compleja de Rey-Osterrieth (V, VI, VII) y tratar de analizar las potencialidades de aquellos que construyen la figura eficazmente (I, II, III). Partiendo de la premisa de que los apoyos deben ser individualizados y moldeados en función de las características y necesidades del individuo, llevar a cabo estudios cualitativos de casos concretos es crucial para potenciar el sistema de apoyos. 


\section{Referencias bibliográficas}

American Psychiatric Association (2014). Manual Diagnóstico y Estadístico de los Trastornos Mentales DSM-5. Panamericana.

Anderson, P. (2002). Assessment and development of executive function (EF) during childhood. Child Neuropsychology, 8(2), pp. 71-82. https://doi.org/10.1076/chin.8.2.71.8724.

Anderson, P. et al. (2001). Assessment and development of organizational ability: The Rey complex figure organizational strategy score (RCF-OSS). The Clinical Neuropsychologist, 15(1), pp. 81-94. https://doi.org/10.1076/ clin.15.1.81.1905.

Ardila, A. et al. (2000). Correlation between intelligence test scores and executive function measures. Archives of Clinical Neuropsychology, 15(1), pp. 31-36. https://doi.org/10.1093/arclin/15.1.31.

Assel, M. A. et al. (2003). Precursors to mathematical skills: examining the roles of visual-spatial skills, executive processes, and parenting factors. Applied Developmental Science, 7(1), pp. 27-38. https://doi.org/10.1207/ s1532480xads0701_3.

Barroso, J. M. y León, J. (2002). Funciones ejecutivas: control, planificación y organización del conocimiento. Revista de Psicología General y Aplicada, 55(1), pp. 27-44. http://hdl.handle.net/11441/51151.

Bigelow, R. T. y Agrawal, Y. (2015). Vestibular involvement in cognition: Visuospatial ability, attention, executive function, and memory. Journal of Vestibular Research, 25(2), pp. 73-89. https://doi.org/10.3233/VES-150544.

Buntinx, W. H. y Schalock, R. L. (2010). Models of disability, quality of life, and individualized supports: Implications for professional practice in intellectual disability. Journal of Policy and Practice in Intellectual Disabilities, 7(4), pp. 283-294. https://doi.org/10.1111/j.1741-1130.2010.00278.x.

Castañeda, N. N. et al. (2017). Funcionamiento ejecutivo en adultos con discapacidad intelectual moderada. Revista Tesis Psicológica, 12(1), pp. 64-82.

Costanzo, F. et al. (2013). Executive functions in intellectual disabilities: A comparison between Williams syndrome and Down syndrome. Research in Developmental Disabilities, 34(5), pp. 1770-1780. https://doi.org/10.1016/j. ridd.2013.01.024.

Danielsson, H. et al. (2010). Executive functions in individuals with intellectual disability. Research in Developmental Disabilities, 31(6), pp. 1299-1304. https://doi.org/10.1016/j.ridd.2010.07.012.

Danielsson, H. et al. (2012). Strengths and weaknesses in executive functioning in children with intellectual disability. Research in Developmental Disabilities, 33(2), pp. 600-607. https://doi.org/10.1016/j.ridd.2011.11.004.

Dias, N. M. y Seabra, A. G. (2012). Executive demands of the Tower of London task in Brazilian teenagers. Psychology \& Neuroscience, 5, pp. 63-75. https://doi.org/10.3922/j.psns.2012.1.09.

Facon, B. y Nuchadee M. L. (2010). An item analysis of Raven's Colored Progressive Matrices among participants with Down syndrome. Research in Developmental Disabilities, 31(1), pp. 243-249. https://doi.org/10.1016/j. ridd.2009.09.011.

Ferguson, H. J. et al. (2021). The developmental trajectories of executive function from adolescence to old age. Scientific reports, 11(1), pp. 1-17. https://doi.org/10.1038/s41598-020-80866-1. 
Friedman, N. P. y Miyake, A. (2017). Unity and diversity of executive functions: Individual differences as a window on cognitive structure. Cortex, 86, pp. 186-204. https://doi.org/10.1016/j.cortex.2016.04.023.

Goyal, S. et al. (2020). Cognitive profile of adults with intellectual disabilities from indigenous communities in Ontario, Canada. Journal of Intellectual \& Developmental Disability, 45(1), pp. 59-65. https://doi.org/10.3109/136 68250.2018.1470160.

Grieco, J. et al. (2015). Down syndrome: Cognitive and behavioral functioning across the lifespan. American Journal of Medical Genetics Part C: Seminars in Medical Genetics, 169(2), pp. 135-149. https://doi.org/10.1002/ ajmg.c.31439.

Harris, J. et al. (2019). Molecularly confirmed Kabuki (Niikawa-Kuroki) syndrome patients demonstrate a specific cognitive profile with extensive visuospatial abnormalities. Journal of Intellectual Disability Research, 63(6), pp. 489-497. https://doi.org/10.1111/jir.12596.

Hung, T. M. et al. (2013). The immediate and sustained effects of acute exercise on planning aspect of executive function. Psychology of Sport and Exercise, 14(5), pp. 728-736. https://doi.org/10.1016/j.psychsport.2013.05.004.

Kitler, P. et al. (2004). Sex difference in performance over 7 years on the Wechsler intelligence scale for children-revised among adult with intellectual disability. Journal of Intellectual Disability Research, 48(2), pp. 114-122. https://doi.org/10.1111/j.1365-2788.2004.00500.x.

Landau, B. y Ferrara K. (2013). Space and language in Williams syndrome: Insights from typical development. Wiley Interdisciplinary Reviews: Cognitive Science, 4, pp. 693-706. https://doi.org/10.1002/wcs.1258.

Lecas, J. F. et al. (2011). Using visual strategies to support verbal comprehension in an adolescent with Down syndrome. Child Language Teaching and Therapy, 27(1), pp. 84-96. https://doi.org/10.1177/0265659010371564.

Lezak, M. D. et al. (2004). Neuropsychological assessment. Oxford University Press.

Loveall, S. J. et al. (2017). A cross-sectional analysis of executive function in Down syndrome from 2 to 35 years. Journal of Intellectual Disability Research, 61(9), pp. 877-887. https://doi.org/10.1111/jir.12396.

Luciana, M. et al. (2009). Tower of London performance in healthy adolescents: The development of planning skills and associations with self-reported inattention and impulsivity. Developmental Neuropsychology, 34(4), pp. 461-475. https://doi.org/10.1080/87565640902964540.

Luzzi, S. et al. (2011). Non-verbal memory measured by Rey-Osterrieth Complex Figure B: normative data. Neurological Sciences, 32(6), pp. 1081-1089. https://doi.org/10.1007/s10072-011-0641-1.

Masson, J. D. et al. (2010). Adaptation and validation of the Tower of London test of planning and problem solving in people with intellectual disabilities. Journal of Intellectual Disability Research, 54(5), pp. 457-467. https://doi. org/10.1111/j.1365-2788.2010.01280.x.

Maulik, P. K. et al. (2011). Prevalence of intellectual disability: a meta-analysis of population-based studies. Research in developmental disabilities, 32(2), pp. 419-436. https://doi.org/10.1016/j.ridd.2010.12.018.

Mervis, C. B. (1999). Visuospatial construction. American journal of human genetics, 65(5), pp. 1222-1229. https:// doi.org/10.1086/302633.

Mervis C. B. et al. (2000). The Williams syndrome cognitive profile. Brain and Cognition, 44, pp. 604-28. https:// doi.org/10.1006/brcg.2000.1232.

Meyers, J. E. y Meyers, K. R. (1995). Rey complex figure test under four different administration procedures. The Clinical Neuropsychologist, 9(1), pp. 63-67. https://doi.org/10.1080/13854049508402059. 
Miyake, A. et al. (2000). The unity and diversity of executive functions and their contributions to complex "Frontal Lobe" tasks: A latent variable analysis. Cognitive Psychology, 41(1), pp. 49-100. https://doi.org/10.1006/ cogp.1999.0734.

Miyake, A. y Friedman, N. P. (2012). The nature and organization of individual differences in executive functions. Current Directions in Psychological Science, 21(1), pp. 8-14. https://doi.org/10.1177/0963721411429458.

Nguyen, N. et al. (2014). Visuospatial anatomy comprehension: The role of spatial visualization ability and problem-solving strategies. Anatomical Sciences Education, 7(4), pp. 280-288. https://doi.org/10.1002/ase.1415.

Nielsen, T. R. y Jørgensen, K. (2013). Visuoconstructional abilities in cognitively healthy illiterate Turkish immigrants: A quantitative and qualitative investigation. The Clinical Neuropsychologist, 27(4), pp. 681-692. https:// doi.org/10.1080/13854046.2013.767379.

Osmon, D. C. et al. (2006). Processing abilities associated with math skills in adult learning disability. Journal of Clinical and Experimental Neuropsychology, 28(1), pp. 84-95. https://doi.org/10.1080/13803390490918129.

Osterrieth, P. (1944). Le teste de copie d'une figure complexe. Archives de Psychologie, 30, pp. 206-356.

Osterrieth, P. (1993). The complex figure copy test. The Clinical Neuropsychologist, 7, pp. 3-21.

Palmqvist, L. et al. (2020). Cognitive abilities and life experience in everyday planning in adolescents with intellectual disabilities: Support for the difference model. Journal of Intellectual Disability Research, 64(3), pp. 209-220. https://doi.org/10.1111/jir.12710.

Pellicano, E. (2010). The development of core cognitive skills in autism: A 3-year prospective study. Child development, 81(5), pp. 1400-1416. https://doi.org/10.1111/j.1467-8624.2010.01481.x.

Pennequin, V. et al. (2010). Motor planning between 4 and 7 years of age: Changes linked to executive functions. Brain and cognition, 74(2), pp. 107-111. https://doi.org/10.1016/j.bandc.2010.07.003.

Raven, J. C. et al. (2001). Raven: matrices progresivas. Escalas color (CPM), general (SPM), superior (APM). TEA Ediciones.

Rey, A. (2009). Test de copia de una figura compleja. TEA Ediciones.

Rowe, J. et al. (2006). Cognitive executive function in Down's syndrome. British Journal of Clinical Psychology, 45(1), pp. 5-17. https://doi.org/10.1348/014466505x29594.

Schalock, R. L. et al. (2007). The renaming of mental retardation: Understanding the change to the term intellectual disability. Intellectual and Developmental Disabilities, 45(2), pp. 116-124. https://doi.org/10.1352/1934-9556(2 007)45[116:TROMRU]2.0.CO;2.

Schalock, R. L. et al. (2021). Intellectual disability. Definition, diagnosis, classification, and systems of supports. $12^{\text {th }}$ ed. American Association on Intellectual and Developmental Disabilities (AAIDD).

Schalock, R. L. y Luckasson, R. (2013). What's at stake in the lives of people with intellectual disability? Part I: The power of naming, defining, diagnosing, classifying, and planning supports. Intellectual and developmental disabilities, 51(2), pp. 86-93. https://doi.org/10.1352/1934-9556-51.2.086.

Shallice, T. (1982). Specific impairments of planning. Philosophical Transcripts of the Royal Society of London, 298, pp. 199-209. https://doi.org/10.1098/rstb.1982.0082.

Shogren, K. A. (2014). The definition of "context" and its application in the field of intellectual disability. Journal of Policy and Practice in Intellectual Disabilities, 11(2), pp. 109-116. https://doi.org/10.1111/jppi.12077. 
Sommers, P. V. (1989). A system for drawing and drawing-related neuropsychology. Cognitive Neuropsychology, 6(2), pp. 117-164. https://doi.org/10.1080/02643298908253416.

Stern, R. A. et al. (1994). The Boston qualitative scoring system for the Rey-Osterrieth complex figure: Description and interrater reliability. The Clinical Neuropsychologist, 8(3), pp. 309-322. https://doi. org/10.1080/13854049408404137.

Tremblay, M. P. et al. (2015). Normative data for the Rey-Osterrieth and the Taylor Complex Figure Tests in Quebec-French people. Archives of Clinical Neuropsychology, 30(1), pp. 78-87. https://doi.org/10.1093/arclin/ acu069.

Troyer, A. y Wishart, H. (1997). A comparison of qualitative scoring systems for the Rey-Osterrieth Complex Figure Test. The Clinical Neuropsychologist, 11(4), pp. 381-390. https://doi.org/10.1080/13854049708400467.

Wehmeyer, M. L. et al. (2008). The intellectual disability construct and its relation to human functioning. Intellectual and Developmental Disabilities, 46(4), pp. 311-318. https://doi.org/10.1352/1934-9556(2008)46[311:TIDCAI]2.0.CO;2. 